\title{
Anisotropic Heat Transfer Inside Rotating Neutron Stars
}

\author{
C. Y. Hui \& K. S. Cheng \\ Department of Physics, The University of Hong Kong, Hong Kong, \\ China
}

\begin{abstract}
We have developed the anisotropic heat transport equation for rotating neutron stars. With a simple model of neutron star, we also model the propagation of heat pulses resulting from transient energy releases inside the star. Even in the slow rotation limit, the results with rotational effects involved could differ significantly from those obtained with a spherically symmetric metric in the timescale of the thermal afterglow.
\end{abstract}

\section{Introduction}

In our study, we have investigated the effects of rotation on the thermal afterglows resulting from transient energy releases inside the star. Three possible forms of energy release, namely, the "shell", "ring" and "spot" cases, are considered. "Shell" is a hypothetical case that energy is released at a particular density in the form of a spherical shell. In the "ring" case, energy is released at a particular density around the rotational equator. This could result from a superfluid-driven glitch (Anderson 1975). In the "spot" case, energy is released in a localized region. This could result from crust-cracking (Ruderman 1969).

\section{2. $\quad$ Model}

The general expression for the line element of an axially symmetric spacetime is determined by time-translational invariance and axial-rotational invariance:

$$
d s^{2}=e^{2 \nu(r, \theta)} d t^{2}-e^{2 \lambda(r, \theta)} d r^{2}-r^{2} e^{2 \psi(r, \theta)}\left[d \theta^{2}+\sin ^{2} \theta(d \phi-\omega(r, \theta) d t)^{2}\right] .
$$

We calculate all the necessary metric functions as well as the stellar structure by using Hartle's formalism (Hartle \& Thorne 1968).

The energy momentum tensor inside a star consisting of a perfect fluid which allows heat flow is:

$$
T^{\mu \nu}=(\rho+P) u^{\mu} u^{\nu}-P g^{\mu \nu}+u^{\mu} q^{\nu}+u^{\nu} q^{\mu} .
$$

With the rotating metric, the vanishing covariant divergence of the energymomentum tensor gives us the heat transport equation:

$$
T_{; \alpha}^{t \alpha}=T_{; t}^{t t}+T_{; r}^{t r}+T_{; \theta}^{t \theta}+T_{; \phi}^{t \phi}=0 .
$$


The equation is checked by recovering to the non-rotational limit (Cheng, Li \& Suen 1998) and the Newtonian limit, with spherically symmetric metric and flat spacetime, respectively.

\section{Monte Carlo Simulations}

The equation of state of neutron matter (Pandharipande 1971) is adopted. We also adopt the heat capacities, thermal conductivity and neutrino emissivities from previous studies (Maxwell 1979; Flowers \& Itoh 1981).

We simulate the propagation of heat pulses by Markovian random walk. Models with different rotational frequencies are compared (see Fig. 1).

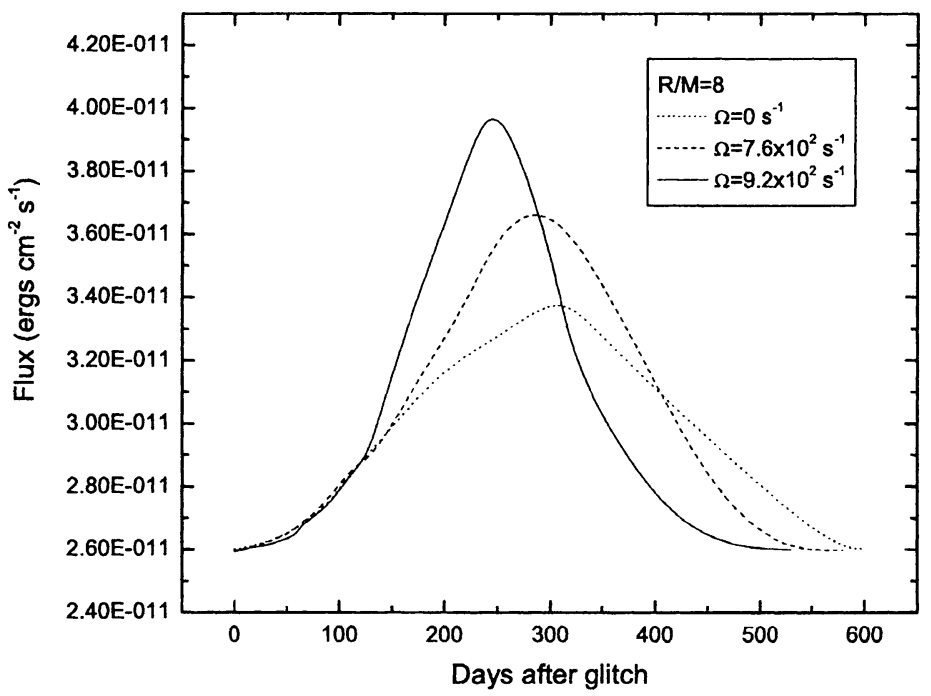

Figure 1. The evolution curves of the flux of thermal X-rays for a neutron star. Three cases with different rotational frequencies are compared.

\section{References}

Anderson, P. 1975, University of Cambridge preprint TCM, 1975/5

Cheng, K. S., Li, Y., \& Suen, W. M. 1998, ApJ, 499, L45

Flowers, E. G., \& Itoh, N. 1981, ApJ, 250, 750

Hartle, J. B., \& Thorne, K. S. 1968, ApJ, 153, 807

Maxwell, O. V. 1979, ApJ, 231, 201

Pandharipande, V. 1971, Nucl. Phys., A174, 641

Ruderman, M. 1969, Nature, 223, 597 